\title{
Infantile hypercalcaemia type 1: a vitamin D-mediated, under-recognised cause of hypercalcaemia
}

\author{
Ryizan Nizar1, Nathan W P Cantley² and Jonathan C Y Tang (D)3 \\ 1Department of Diabetes and Endocrinology, 2Department of Clinical Biochemistry, Southmead Hospital, North \\ Bristol NHS Trust, Bristol, UK, and ${ }^{3}$ Norwich Medical School, University of East Anglia, Norwich Research Park, \\ Norwich, UK
}

Correspondence should be addressed to N W P Cantley Email

nathan.cantley@nhs.net

\section{Summary}

A 33-year-old gentleman of Egyptian heritage presented with a 21 years history of unexplained and recurrent hypercalcaemia, nephrolithiasis, nephrocalcinosis, and myocarditis. A similar history was also found in two first-degree relatives. Further investigation into the vitamin $D$ metabolism pathway identified the biochemical hallmarks of infantile hypercalcaemia type $1(\mathrm{IHH})$. A homozygous, likely pathogenic, variant in CYP24A1 was found on molecular genetic analysis confirming the diagnosis. Management now focuses on removing excess vitamin D from the metabolic pathway as well as reducing calcium intake to achieve serum-adjusted calcium to the middle of the reference range. If undiagnosed, IIH can cause serious renal complications and metabolic bone disease.

\section{Learning points:}

- Infantile hypercalcaemia type $1(\mathrm{IIH})$ is an autosomal recessive disorder characterised by homozygous mutations in the CYP24A1 gene that encodes the 24-hydroxylase enzyme used to convert active vitamin D metabolites such as 1,25-(OH) 2 -vitamin D into their inactive form.

- IIH should be questioned in individuals presenting with a history of unexplained hypercalcaemia, especially if presenting from childhood and/or where there is an accompanying family history of the same in first and/or second degree relatives, causing complications such as nephrocalcinosis, pericarditis, and calcium-based nephrolithiasis.

- Associated biochemistry of IIH is persistent mild to moderate hypercalcaemia, normal or raised 25-(OH)-vitamin D and elevated 1,25-(OH $)_{2}$-vitamin D. An elevated ratio of 25-(OH)-vitamin D to 24,25-(OH) $)_{2}$-vitamin D can be a useful marker of defects in the 24-hydroxylase enzyme, whose measurement can be facilitated through the supraregional assay service.

- Management should focus on limiting the amount of vitamin D introduced into the body either via sunlight exposure or supplementation in addition to calcium dietary restriction to try and maintain appropriate calcium homeostasis

\section{Background}

Vitamin D excess, either as a result of excess intake or reduced inactivation/excretion, is recognised to be a cause of hypercalcaemia, although seldom considered. Subtle elevations in the $25-(\mathrm{OH})$ and $1,25-(\mathrm{OH})_{2}$ forms of vitamin $\mathrm{D}$ can go unnoticed and be poorly understood.
The condition we describe here is under-recognised in clinical practice. When these individuals are eventually diagnosed as adults, they commonly are found to have had biochemical/pathological features extending back from childhood (1). We present this case to provide further education and prevent other patients from undergoing the same significant delay in appropriate diagnosis. 


\section{Case presentation}

A 33-year-old man of Egyptian heritage incidentally came to the attention of endocrinology during an admission to acute medicine presenting with symptoms of chest pain thought to be the 8th presentation in 4 years with myocarditis-associated symptoms. Cause for the myocarditis was documented in cardiology outpatient letters as being 'undetermined'. The patient did not have any other classical features on history or examination associated with hypercalcaemia on this presentation.

The patient had an extensive past medical history of renal calculi - first presenting at age 12 while still living in Egypt. Subsequent repeat renal calculi developed at age 16 and 17. The patient had an otherwise normal development history since his childhood. He then presented to urology services in the UK as an adult due to hydronephrosis secondary to renal calculi. Both brother and father of this patient also suffered from nephrolithiasis indicating a possible genetic aetiology to the shared symptoms. The patient had also been reviewed in a metabolic renal stone clinic.

The patient did not have specific findings on clinical examination aside from the symptoms associated with the myocarditis of chest pain and shortness of breath, and renal angle abdominal pain whenever renal calculi arose.

\section{Investigation}

A summary of biochemical and radiological investigations is documented in Table 1. Other investigations performed include renal calculus analysis by infrared spectroscopy in 2009 which revealed $98 \%$ content of the extracted calculus was calcium phosphate. Repeated oxalate and citrate output demonstrated results either within the reference range or only mildly elevated (citrate output: $1.4 \mathrm{mmol} / 24 \mathrm{~h}$ (reference range (RR): $1.3-6.0 \mathrm{mmol} / 24 \mathrm{~h}$ ), oxalate output: $380 \mu \mathrm{mol} / 24 \mathrm{~h}$ (RR: $<460 \mu \mathrm{mol} / 24 \mathrm{~h}$ )). Fibroblast growth factor-23 (FGF-23) and retinol binding protein (RBP) levels were checked historically as part of his initial work up in 2011 and were within the reference range when measured $(\mathrm{RBP} /$ creatinine ratio $5.0 \mu \mathrm{g} / \mathrm{mmol}$ creatinine (RR: $<18 \mu \mathrm{g} / \mathrm{mmol}$ creatinine), FGF-23: $93 \mathrm{RU} / \mathrm{mL}$ (RR: < $100 \mathrm{RU} / \mathrm{mL}$ )). FGF-23 is one of the most important regulators of serum phosphate and 1,25-(OH $)_{2}$-vitamin D levels. High levels of FGF-23 suppress the activity of $1-\alpha$ hydroxylase activity leading to low 1,25-(OH) $)_{2}$-vitamin D levels (2). However FGF-23 deficiency or resistance can lead to a state of high 1,25- $(\mathrm{OH})_{2}$-vitamin D levels with high phosphate levels. There are hereditary and acquired forms of FGF-23 deficiency/resistance and this manifests with tumoural calcinosis (2). RBP is used by renal physicians to rule out renal tubular injury as a cause of underlying renal disease. This patient had been followed-up by the renal team for a number of years for unexplained nephrocalcinosis and he would have had a RBP level checked initially to rule out renal tubular damage.

As part of this patients hypercalcaemia work-up, he also had a DEXA scan that showed consistent signs of osteopenia (T-score between -1 and -2.5 ) within the lumbar spine region.

In June 2020, a 24,25-(OH) ${ }_{2}$-vitamin D level measured at Norwich and Norfolk Hospital Supra-regional assay lab returned a result of $1.2 \mathrm{nmol} / \mathrm{L}$ (RR: $1.1-13.5 \mathrm{nmol} / \mathrm{L}$ ). With an accompanying 25-OH-vitamin D of $79 \mathrm{nmol} / \mathrm{L}$,

Table 1 Summary of historical patient investigations - results described with accompanying reference ranges.

\begin{tabular}{|c|c|c|c|c|c|c|c|}
\hline Analyte & $\begin{array}{c}\text { Reference } \\
\text { range }\end{array}$ & 2009-2010 & 2011 & 2014 & 2016 & 2019 & 2020 \\
\hline Phosphate (mmol/L) & $0.85-1.40$ & 1.00 & 0.75 & 1.08 & 1.06 & 1.21 & 1.04 \\
\hline Calcium (mmol/L) & $2.2-2.6$ & 2.39 & 2.39 & 2.71 & 2.59 & 2.74 & 2.64 \\
\hline Adjusted calcium (mmol/L) & $2.2-2.6$ & 2.54 & 2.47 & 2.77 & 2.63 & 2.69 & 2.64 \\
\hline Creatinine $(\mu \mathrm{mol} / \mathrm{L})$ & $60-110$ & 90 & 95 & 101 & 103 & 103 & 103 \\
\hline eGFR (mL/min/1.73 m) & $>60$ & 90 & 84 & 77 & 74 & 82 & 82 \\
\hline Magnesium (mmol/L) & $0.66-1.07$ & 0.72 & 0.70 & 0.80 & & 0.75 & 0.75 \\
\hline Parathyroid hormone (nmol/L) & $1.6-6.9$ & 1.40 & 1.20 & 1.00 & 1.00 & 0.70 & 0.80 \\
\hline 25-OH-vitamin D (nmol/L) & $>70$ & 58.0 & 41.0 & 54.0 & 38.0 & 55 & 57 \\
\hline $1,25-(\mathrm{OH})_{2}-$ Vitamin D (pmol/L) & $40-150$ & 159.0 & 112 & & & & 153 \\
\hline Urine calcium (mmol/L) & & 5.51 & & 3.70 & 4.23 & & 5.95 \\
\hline $24 \mathrm{~h}$ urine calcium (mmol/24 h) & & 7.30 & & 5.10 & 6.60 & & 8.4 \\
\hline Calcium/creatinine ratio ( $<0.5 \mathrm{mmol} / \mathrm{mmol}$ creatinine) & & 0.57 & & 0.52 & 0.423 & & 0.52 \\
\hline CT renal & & $\begin{array}{l}\text { Bilateral } \\
\text { calculi* }\end{array}$ & & Medullary NC & & $\begin{array}{l}\text { R Renal } \\
\text { calculi, NC }\end{array}$ & \\
\hline DEXA T-score lumbar spine & & & -1.7 & -1.5 & & -1.7 & \\
\hline
\end{tabular}


this yielded a significantly elevated 25-OH-vitamin D to $24,25-(\mathrm{OH})_{2}$-vitamin $\mathrm{D}$ ratio of 66 (upper limit of normal=23). A mildly elevated $1,25-(\mathrm{OH})_{2}$-vitamin D (153 pmol/L (RR: 55-139 nmol/L)) means the ratio of 1,25- $(\mathrm{OH})_{2}$-vitamin $\mathrm{D}$ to $24,25-(\mathrm{OH})_{2}$-vitamin $\mathrm{D}$ was extremely high at 128, a further feature of IIH. Genetic analysis by next-generation sequencing of CYP24A1 revealed a biallelic 3 bp deletion at codon 143 (Glu143del) that has a deleterious effect on the enzyme and classified as a class 4 variant (likely pathogenic) (3). Given both biochemical and genetic hallmarks were concordant - a diagnosis of IIH was made.

\section{Treatment}

Prior to the diagnosis, the patient was prescribed: indapamide $2.5 \mathrm{mg}$ once daily, nebivolol $2.5 \mathrm{mg}$ twice daily. The treatment plan for this patient following diagnosis aimed to reduce his intake of dietary calcium as well as any medications which could exacerbate hypercalcaemia. Additionally, exogenous sources of vitamin D which could exacerbate his $1,25-(\mathrm{OH})_{2}$-vitamin $\mathrm{D}$ were removed. The summary of the initial treatment plan was as follows:

- Avoid all exogenous vitamin D supplementation.

- Avoid prolonged sun exposure which may increase the production of active vitamin D.

- Reduce dietary calcium intake titrating this against achieving serum calcium within the middle of the reference range $(2.2-2.6 \mathrm{mmol} / \mathrm{L})$.

If this failed to correct his serum calcium then the next treatment trial would be oral fluconazole. Acute presentations with markedly elevated serum calcium levels could be treated with short courses of prednisolone to inhibit 1,25-(OH) ${ }_{2-}$ vitamin D levels.

\section{Outcome and follow-up}

As a first step, renal dietetic input will start working to achieve a low calcium diet and instructions to care providers for the patient to not introduce any exogenous sources of vitamin D. At the time of manuscript submission, this has been slow to initiate, but regular follow-up is planned on diet adherence with repeated biochemistry, to identify if next-line therapy (trial of fluconazole) is indicated. Other first-degree relatives of the patient have been advised to be screened for the same mutation to confirm the carrier/affected status of the parents and siblings, respectively.

\section{Discussion}

$\mathrm{IIH}$ is a rare condition with its prevalence in the general population unknown (4). In the UK, there had been concerns of vitamin D induced hypercalcaemia in some children as far back as the 1950s. Interestingly, this was the same time at which vitamin D supplementation or food fortification was introduced to prevent Rickets disease. There were 204 reported cases within a 2-year period up to July 1955 (1). In the vast majority, it was thought to be due to high intake of vitamin $\mathrm{D}$. This would suggest that disorders of vitamin D metabolism are more prevalent but only some present with symptoms due to phenotypic variability (5).

Infantile hypercalcaemia is not a 'benign' condition as initially postulated and in our patient, he experienced nephrocalcinosis, osteopenia, GI symptoms, and pericarditis (3).

There are two known causative genes/'types' of infantile hypercalcaemia. Type 1 is caused by loss-offunction mutation in CYP24A1 while type 2 is caused by mutation in SLC34A1 gene. Mutation in CYP24A1 leads to the phenotype we describe here (Fig. 1). Mutation in SLC34A1 reduces serum phosphate levels due to a defect in sodium-dependant phosphate transporters, leading to low body stores of phosphates, and for which more vitamin D is activated to compensate.

Pragmatically for clinical practice, a pattern of high calcium, low PTH in a young person with no concerns of malignancy should prompt to check 1,25-(OH $)_{2}^{-}$ vitamin $\mathrm{D}$ level in addition to 25-OH-vitamin D. An inappropriately high $1,25-(\mathrm{OH})_{2}$-vitamin D levels should prompt reflection on possible causes related to vitamin D metabolism and further testing of other vitamin D metabolites. Persistently, a high level of 1,25-(OH $)_{2}^{-}$ vitamin $\mathrm{D}$ leads to increased intestinal absorption of calcium as well as increased bone resorption. This sustained bone resorption leads to the osteopenia/ osteoporosis described in this case (6).

Measurement of $24,25-(\mathrm{OH})_{2}$-vitamin D is available to request through the supra-regional vitamin $\mathrm{D}$ assay service at Norwich and Norfolk Hospitals in partnership with the University of East Anglia (7). This uses a liquid-chromatography-tandem mass spectroscopy (LC-MS/MS) method and provides a level of 24,25-(OH $)_{2}$ vitamin $\mathrm{D}$ as well as a ratio of $25-(\mathrm{OH})$-vitamin $\mathrm{D}$ to 24,25-(OH $)_{2}$-vitamin D. Sample requirements for $24,25-(\mathrm{OH})_{2}$-vitamin $\mathrm{D}$ are $100 \mu \mathrm{L}$ of separated serum sent at room temperature. The ratio of $25-(\mathrm{OH})$-vitamin $\mathrm{D}$ to 24,25 - $(\mathrm{OH})_{2}$-vitamin $\mathrm{D}$ has a cut-off of $>23$ for being 


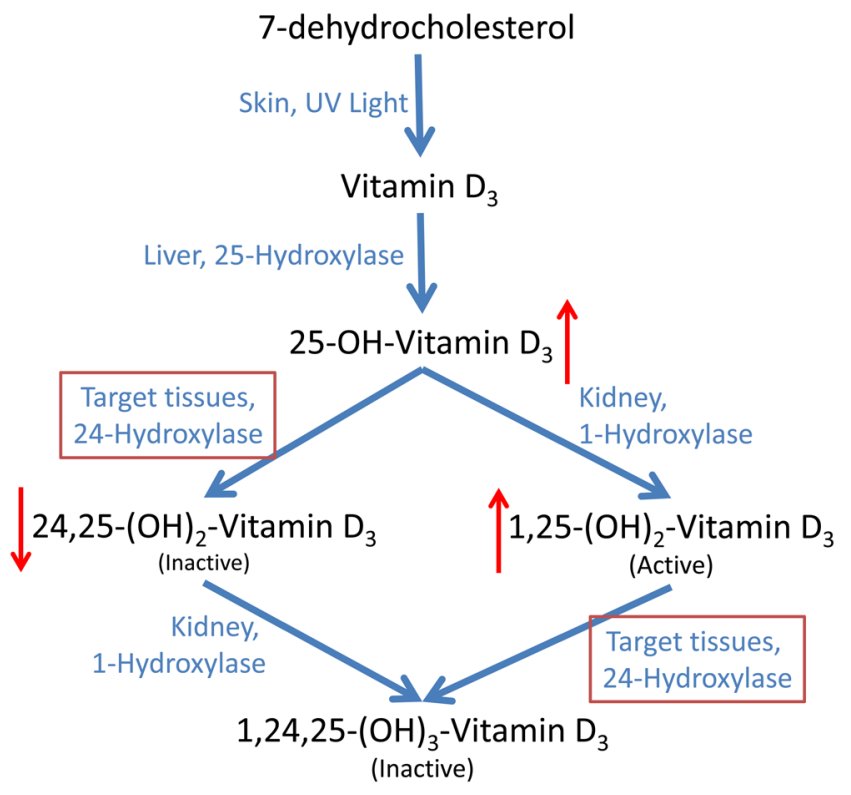

Figure 1

A summary of the vitamin D metabolic pathway including enzymes and location of activity. The enzyme affected by $\mathrm{IH}$ is highlighted by a red box. Red arrows indicate the expected changes in measurable vitamin $D$ metabolites in IIH. Adapted from (A), Tebben et al. (4).

associated with altered vitamin D status (8) Ultimately, the diagnosis is confirmed by identifying biallelic pathogenic variants. The Online Mendelian Inheritance in Man (OMIM) catalogue describes a range of mutations found in literature to cause IIH, including the Glu143del variant found in our patient (5). CYP24A1 testing is part of the NHS England National Genomic Testing Directory within panel R256 for nephrolithiasis (9).

Treatment of this condition is potentially simple to initiate but can be challenging to maintain. Primarily treatment should be aimed at reducing vitamin D and calcium intake in addition to avoid prolonged sun exposure. Short courses of steroids can be used; however, in the long term, this is not feasible and there are case reports of successful use of fluconazole given this inhibits the 1 - $\alpha$-hydroxylase enzyme (10).

\section{Declaration of interest}

The authors declare that there is no conflict of interest that could be perceived as prejudicing the impartiality of the research reported.

\section{Funding}

This work did not receive any specific grant from any funding agency in the public, commercial or not-for-profit sector.

\section{Patient consent}

$\mathrm{R} \mathrm{N}$ discussed publication of this case directly with the patient via telephone appointment. Written signed consent was gained from the patient for publication of the submitted article and accompanying images.

\section{Author contribution statement}

$\mathrm{NC}$ contributed to arrange further vitamin $\mathrm{D}$ testing with referral laboratory and to the writing of the manuscript. J T contributed background data on vitamin $\mathrm{D}$ metabolite analysis. $\mathrm{R} \mathrm{N}$ contributed to direct care of the patient and writing of the manuscript.

\section{References}

1 Hypercalcaemia in infants and vitamin D. BMJ 19562 149-149. (https://doi.org/10.1136/bmj.2.4985.149)

2 Liu S \& Quarles LD. How fibroblast growth factor 23 works. Journal of the American Society of Nephrology 200718 1637-1647. (https://doi. org/10.1681/ASN.2007010068)

3 Schlingmann KP, Kaufmann M, Weber S, Irwin A, Goos C, Ulrike J, Misselwitz J, Klaus G, Kuwertz-Broking E, Fehrenbach H, et al. Mutations in CYP24A1 and idiopathic infantile hypercalcemia. New England Journal of Medicine 2001365 410-421. (https://doi. org/10.1056/NEJMoa1103864)

4 Tebben PJ, Singh RJ \& Kumar R. Vitamin D-mediated hypercalcemia: mechanisms, diagnosis, and treatment. Endocrine Reviews 201637 521-547. (https://doi.org/10.1210/er.2016-1070)

5 O'Neil MJF. Hypercalcaemia, infantile Type 1 [internet]. OMIM 2011. (available at: https://www.omim.org/entry/143880)

6 De Paolis E, Scaglione GL, De Bonis M, Minucci A \& Capoluongo E. CYP24A1 and SLC34A1 genetic defects associated with idiopathic infantile hypercalcemia: from genotype to phenotype. Clinical Chemistry and Laboratory Medicine 201957 1650-1667. (https://doi. org/10.1515/cclm-2018-1208)

7 Supra-Regional Assay Service. Norfolk and Norwich University Hospital bone marker service [internet], 2020. (available at: http:// www.sas-centre.org/centres/bone-markers/norwich)

8 Tang JCY, Nicholls H, Piec I, Washbourne CJ, Dutton JJ, Jackson S, Greeves J \& Fraser WD. Reference intervals for serum 24,25-dihydroxyvitamin D and the ratio with 25-hydroxyvitamin D established using a newly developed LC-MS/MS method. Journal of Nutritional Biochemistry 201746 21-29. (https://doi.org/10.1016/j. jnutbio.2017.04.005)

9 NHS England. National Genomic Test Directory for Rare and Inherited Disease. NHS England, 2020. (available at: https://www.england. nhs.uk/wp-content/uploads/2018/08/Rare-and-Inherited-DiseaseEligibility-Criteria-November-2020-21.pdf)

10 Sayers J, Hynes AM, Srivastava S, Dowen F, Quinton R, Darra HK \& Sayer JA. Successful treatment of hypercalcaemia associated with a CYP24A1 mutation with fluconazole. Clinical Kidney Journal 20158 453-455. (https://doi.org/10.1093/ckj/sfv028) 